Bull. Korean Math. Soc. 46 (2009), No. 5, pp. 967-978

DOI 10.4134/BKMS.2009.46.5.967

\title{
A STUDY ON RECTIFYING CURVES IN THE DUAL LORENTZIAN SPACE
}

\author{
Emine Özbey and Mehmet Oral
}

\begin{abstract}
In this work, we give some characterizations of rectifying curves in dual Lorentzian space. Also, we show that rectifying dual Lorentzian curves can be stated by the aid of dual unit spherical curves.
\end{abstract}

\section{Introduction}

Dual numbers were introduced by W. K. Clifford (1849-1879) as a tool for his geometrical investigations. After him E. Study used dual numbers and dual vectors in his research on the geometry of lines and kinematics. He devoted special attention to the representation of directed lines by dual unit vectors and defined the mapping that is known by his name. There exists one-to-one correspondence between the points of dual unit sphere $\mathbb{S}^{2}$ and the directed lines in $\mathbb{R}^{3}[5]$.

If we take the Minkowski 3-space $\mathbb{R}_{1}^{3}$ instead of $\mathbb{R}^{3}$ the E. Study mapping can be stated as follows: The dual timelike and spacelike unit vectors of dual hyperbolic and Lorentzian unit spheres $\mathbb{H}_{0}^{2}$ and $\mathbb{S}_{1}^{2}$ at the dual Lorentzian space $\mathbb{D}_{1}^{3}$ are in one-to-one correspondence with the directed timelike and spacelike lines in $\mathbb{R}_{1}^{3}$, respectively. Then a differentiable curve on $\mathbb{H}_{0}^{2}$ corresponds to a timelike ruled surface at $\mathbb{R}_{1}^{3}$. Similarly, the timelike (resp. spacelike) curve on $\mathbb{S}_{1}^{2}$ corresponds to any spacelike (resp. timelike) ruled surface in $\mathbb{R}_{1}^{3}[8]$.

We will survey briefly the fundamental concepts and properties in the Lorentzian space. We refer mainly to O'Neill [7].

Let $\mathbb{R}_{1}^{3}$ be the 3 -dimensional the Lorentzian space with Lorentzian metric $\langle\rangle=,-d x_{1}^{2}+d x_{2}^{2}+d x_{3}^{2}$. It has been known that in $\mathbb{R}_{1}^{3}$ there are three categories of curves and vectors, namely, spacelike, timelike and null, depending on their causal character. Let $\vec{x}$ be a tangent vector of Lorentzian space. Then $\vec{x}$ is said to be spacelike if $\langle\vec{x}, \vec{x}\rangle>0$ or $\vec{x}=\overrightarrow{0}$, timelike if $\langle\vec{x}, \vec{x}\rangle<0$, null (lightlike) if $\langle\vec{x}, \vec{x}\rangle=0$ and $\vec{x} \neq \overrightarrow{0}$. Let $\alpha: I \subset \mathbb{R} \rightarrow \mathbb{R}_{1}^{3}$ be a regular curve

Received August 18, 2008.

2000 Mathematics Subject Classification. 53A05, 53A35,53C50.

Key words and phrases. dual Lorentzian space, rectifying dual Lorentzian curve, Frenet formulae, dual Darboux vector. 
in $\mathbb{R}_{1}^{3}$. Then, the curve $\alpha$ is spacelike if all its velocity vectors are spacelike. Similarly, it is called timelike and null curve if all its velocity vectors are timelike and null vectors, respectively.

A dual number $\hat{x}$ has the form $x+\varepsilon x^{*}$ with properties

$$
\varepsilon \neq 0, \quad 0 \varepsilon=\varepsilon 0=0, \quad 1 \varepsilon=\varepsilon 1=\varepsilon, \quad \varepsilon^{2}=0,
$$

where $x$ and $x^{*}$ are real numbers and $\varepsilon$ is the dual unit (for the properties of dual numbers, see [9]). An ordered triple of dual numbers $\left(\hat{x}_{1}, \hat{x}_{2}, \hat{x}_{3}\right)$ is called a dual vector and the set of dual vectors is denoted by

$$
\begin{aligned}
\mathbb{D}^{3}=\mathbb{D} \times \mathbb{D} \times \mathbb{D}=\{\overrightarrow{\hat{x}} \mid \overrightarrow{\hat{x}} & =\left(x_{1}+\varepsilon x_{1}^{*}, x_{2}+\varepsilon x_{2}^{*}, x_{3}+\varepsilon x_{3}^{*}\right) \\
& =\left(x_{1}, x_{2}, x_{3}\right)+\varepsilon\left(x_{1}^{*}, x_{2}^{*}, x_{3}^{*}\right) \\
& \left.=\vec{x}+\varepsilon \overrightarrow{x^{*}}, \quad \vec{x}, \overrightarrow{x^{*}} \in \mathbb{R}^{3}\right\} .
\end{aligned}
$$

For any $\vec{x}=\vec{x}+\varepsilon \overrightarrow{x^{*}}, \vec{y}=\vec{y}+\varepsilon \overrightarrow{y^{*}} \in \mathbb{D}^{3}$, if the Lorentzian inner product of dual vectors $\vec{x}$ and $\overrightarrow{\hat{y}}$ is defined by

$$
\langle\overrightarrow{\hat{x}}, \overrightarrow{\hat{y}}\rangle=\langle\vec{x}, \vec{y}\rangle+\varepsilon\left(\left\langle\vec{x}, \overrightarrow{y^{*}}\right\rangle+\left\langle\overrightarrow{x^{*}}, \vec{y}\right\rangle\right),
$$

then the dual space $\mathbb{D}^{3}$ together with this Lorentzian inner product is called as dual Lorentzian space and it is shown by $\mathbb{D}_{1}^{3}$. A dual vector $\vec{x}$ in $\mathbb{D}_{1}^{3}$ is said to be spacelike, timelike and lightlike (null) if the vector $\vec{x}$ is spacelike, timelike and lightlike (null), respectively. Lorentzian vectorial product of dual vectors $\overrightarrow{\hat{x}}=\left(\hat{x}_{1}, \hat{x}_{2}, \hat{x}_{3}\right)$ and $\overrightarrow{\hat{y}}=\left(\hat{y}_{1}, \hat{y}_{2}, \hat{y}_{3}\right)$ in $\mathbb{D}_{1}^{3}$ is defined by

$$
\overrightarrow{\hat{x}} \wedge \overrightarrow{\hat{y}}=\left(\hat{x}_{3} \hat{y}_{2}-\hat{x}_{2} \hat{y}_{3}, \hat{x}_{3} \hat{y}_{1}-\hat{x}_{1} \hat{y}_{3}, \hat{x}_{1} \hat{y}_{2}-\hat{x}_{2} \hat{y}_{1}\right) \text {. }
$$

If $\vec{x} \neq 0$, the norm $\|\vec{x}\|$ of $\vec{x}=\vec{x}+\varepsilon \overrightarrow{x^{*}}$ is defined by

$$
\|\overrightarrow{\hat{x}}\|=\sqrt{|\langle\overrightarrow{\hat{x}}, \overrightarrow{\hat{x}}\rangle|}
$$

A dual vector $\vec{x}$ with norm 1 is called a dual unit vector. Let $\vec{x}=\vec{x}+\varepsilon \overrightarrow{x^{*}} \in$ $\mathbb{D}_{1}^{3}$. Then,

i) The set

$\mathbb{S}_{1}^{2}=\left\{\overrightarrow{\hat{x}}=\vec{x}+\varepsilon \overrightarrow{x^{*}} \mid\|\overrightarrow{\hat{x}}\|=(1,0) ; \vec{x}, \overrightarrow{x^{*}} \in \mathbb{R}_{1}^{3}\right.$ and the vector $\vec{x}$ is spacelike $\}$

is called the pseudo dual sphere with the center $\hat{O}$ in $\mathbb{D}_{1}^{3}$.

ii) The set

$\mathbb{H}_{0}^{2}=\left\{\overrightarrow{\hat{x}}=\vec{x}+\varepsilon \overrightarrow{x^{*}} \mid\|\overrightarrow{\hat{x}}\|=(1,0) ; \vec{x}, \overrightarrow{x^{*}} \in \mathbb{R}_{1}^{3}\right.$ and the vector $\vec{x}$ is timelike $\}$ is called the pseudo dual hyperbolic space in $\mathbb{D}_{1}^{3}[8]$. 
If every real valued functions $x_{i}(t)$ and $x_{i}^{*}(t), 1 \leq i \leq 3$, are differentiable, dual Lorentzian curve

$$
\begin{aligned}
\hat{x}: I \subset \mathbb{R} & \rightarrow \underset{\mathbb{D}_{1}^{3}}{\overrightarrow{3}} \\
t \rightarrow \hat{x}(t) & =\left(\underset{x_{1}(t)+\varepsilon x_{1}^{*}}{\vec{\longrightarrow}}(t), x_{2}(t)+\varepsilon x_{2}^{*}(t), x_{3}(t)+\varepsilon x_{3}^{*}(t)\right) \\
& =x(t)+\varepsilon x^{*}(t)
\end{aligned}
$$

in $\mathbb{D}_{1}^{3}$ is differentiable. We call the real part $\overrightarrow{x(t)}$ as indicatrix of $\vec{x}(t)$. The dual arc length of the curve $\hat{x}(t)$ from $t_{1}$ to $t$ is defined as

$$
\hat{s}=\int_{t_{1}}^{t}\left\|\hat{x}(t){ }^{\prime}\right\| d t=\int_{t_{1}}^{t}\left\|\overrightarrow{x(t)}{ }^{\prime}\right\| d t+\varepsilon \int_{t_{1}}^{t}\left\langle\vec{t}, x^{*}(t)^{\prime}\right\rangle d t=s+\varepsilon s^{*},
$$

where $\vec{t}$ is a unit tangent vector of $\overrightarrow{x(t)}$. From now on we will take the arc length $s$ of $\overrightarrow{x(t)}$ as the parameter instead of $t$.

The equalities relative to derivatives of dual Frenet vectors $\vec{t}, \overrightarrow{\hat{n}}, \overrightarrow{\hat{b}}$ throughout the dual Lorentzian curve are written in the matrix form

$$
\frac{d}{d \hat{s}}\left[\begin{array}{c}
\overrightarrow{\hat{t}} \\
\overrightarrow{\hat{n}} \\
\overrightarrow{\hat{b}}
\end{array}\right]=\left[\begin{array}{ccc}
0 & \hat{\kappa} & 0 \\
-\epsilon_{1} \epsilon_{2} \hat{\kappa} & 0 & \hat{\tau} \\
0 & -\epsilon_{2} \epsilon_{3} \hat{\tau} & 0
\end{array}\right]\left[\begin{array}{c}
\overrightarrow{\hat{t}} \\
\overrightarrow{\hat{n}} \\
\overrightarrow{\hat{b}}
\end{array}\right],
$$

where $\hat{\kappa}=\kappa+\varepsilon \kappa^{*}$ is nowhere pure dual curvature and $\hat{\tau}=\tau+\varepsilon \tau^{*}$ is nowhere pure dual torsion and $\langle\overrightarrow{\hat{t}}, \overrightarrow{\hat{t}}\rangle=\epsilon_{1},\langle\overrightarrow{\hat{n}}, \overrightarrow{\hat{n}}\rangle=\epsilon_{2},\langle\overrightarrow{\hat{b}}, \overrightarrow{\hat{b}}\rangle=\epsilon_{3},\langle\overrightarrow{\hat{t}}, \overrightarrow{\hat{n}}\rangle=\langle\overrightarrow{\hat{t}}, \overrightarrow{\hat{b}}\rangle=$ $\langle\overrightarrow{\hat{b}}, \overrightarrow{\hat{n}}\rangle=0$. The formulae (1.2) are called the Frenet formulae in the dual Lorentzian space (see [1], [2] and [12] for details). The planes spanned by $\{\overrightarrow{\hat{t}}, \overrightarrow{\hat{b}}\},\{\overrightarrow{\hat{t}}, \overrightarrow{\hat{n}}\}$, and $\{\overrightarrow{\hat{n}}, \overrightarrow{\hat{b}}\}$ at each point of the dual Lorentzian curve are called the rectifying plane, the osculating plane, and the normal plane, respectively.

When the position vector of a dual Lorentzian curve $\vec{x}(s)$ lies in the rectifying plane its which is called rectifying dual Lorentzian curve. Therefore, the position vector of $\hat{x}(s)$ can be written as

$$
\hat{x}(s)=\hat{\lambda}(s) \hat{t}(s)+\hat{\mu}(s) \hat{b}(s)
$$

for some dual functions $\hat{\lambda}(s)$ and $\hat{\mu}(s)$.

A useful method of determining rectifying curves in the Euclidean 3-dimensional space $\mathbb{R}^{3}$ has been developed by Chen [3]. He shows that it can be possible to determine completely all rectifying curves in $\mathbb{R}^{3}$. In [6], the authors have characterized non-null and null rectifying curves lying fully in the Minkowski 
3-space. Yücesan, Ayyıldız, and Çöken have given some characterizations of the rectifying curves in the dual space $\mathbb{D}^{3}[11]$.

By using this method, we have characterizations of rectifying curves in the dual Lorentzian space $\mathbb{D}_{1}^{3}$. In Section 2, we give characterizations of rectifying curves in $\mathbb{D}_{1}^{3}$. In Section 3, we characterize rectifying curves with respect to the distance function and the components of the position vector of dual Lorentzian curve. Also we get characterization of rectifying dual Lorentzian curves in terms of its harmonic curvature.

In the last section, we show that rectifying dual Lorentzian space curves can be stated by the aid of dual unit spherical curves.

Therefore, this work gives a link with the classical surface theory since a differentiable curve on $\mathbb{H}_{0}^{2}$ corresponds to a timelike ruled surface in $\mathbb{R}_{1}^{3}$ and similarly the timelike (resp. spacelike) curve on $\mathbb{S}_{1}^{2}$ corresponds to any spacelike (resp. timelike) ruled surface in $\mathbb{R}_{1}^{3}[10]$.

\section{Some characterizations of rectifying curves in $\mathbb{D}_{1}^{3}$}

In this section, we shall give two theorems relative to properties of rectifying curves in dual Lorentzian space $\mathbb{D}_{1}^{3}$.

Theorem 2.1. Let $\hat{x}: I \rightarrow \mathbb{D}_{1}^{3}$ be a dual timelike or dual spacelike rectifying curve in dual Lorentzian space $\mathbb{D}_{1}^{3}$ with $\hat{\kappa}=\kappa+\varepsilon \kappa^{*}$ such that $\kappa>0$ and let $\hat{s}$ be dual arc length of the dual Lorentzian curve $\hat{x}$. Then the following statements hold:

(i) The dual distance function $\hat{\rho}=\|\hat{x}(s)\|$ satisfies $\hat{\rho}^{2}=\left|\epsilon_{1} \hat{s}^{2}+\hat{c}_{1} \hat{s}+\hat{c}_{2}\right|$ for some dual constants $\hat{c}_{1}$ and $\hat{c}_{2}$.

(ii) The tangential component of the position vector of the dual Lorentzian curve is given by

$$
\langle\hat{x}(\vec{s}), \hat{t}(s)\rangle=\epsilon_{1} \hat{s}+\hat{k},
$$

where $\hat{k}$ is a dual constant.

(iii) The normal component $\hat{x}^{N}$ of the position vector of the dual Lorentzian curve is of dual constant length and the dual distance function $\hat{\rho}$ is non-constant.

(iv) The torsion $\hat{\tau}=\tau+\varepsilon \tau^{*}$ is nowhere pure dual that is $\tau \neq 0$, and the binormal component $\langle\hat{x}(\vec{s}), \hat{b}(s)\rangle$ of the position vector is dual constant.

Conversely, if $\hat{x}: I \rightarrow \mathbb{D}_{1}^{3}$ is a dual timelike or a dual spacelike curve in $\mathbb{D}_{1}^{3}$ with $\hat{\kappa}=\kappa+\varepsilon \kappa^{*}$, where $\kappa>0$, and if one of (i), (ii), (iii), or (iv) holds, then $\hat{x}$ is a rectifying dual Lorentzian curve.

Proof. Let us first suppose that $\hat{x}: I \rightarrow \mathbb{D}_{1}^{3}$ is a non-null dual rectifying curve parametrized by the dual arc length function $\hat{s}$. Then the position vector $\vec{x}(s)$ of the dual Lorentzian curve $\hat{x}$ satisfies the equation

$$
\hat{x}(s)=\hat{\lambda}(s) \hat{t}(s)+\hat{\mu}(s) \hat{b}(s)
$$


for some dual functions $\hat{\lambda}(s)$ and $\hat{\mu}(s)$. Differentiating equation (2.1) with respect to the dual arc length function $\hat{s}$ and by using the Frenet equations (1.2), we get

$$
\frac{d}{d \hat{s}} \hat{\lambda}(s)=(1,0)=1+\varepsilon 0=1, \quad \hat{\lambda}(s) \hat{\kappa}(s)=\epsilon_{2} \epsilon_{3} \hat{\tau}(s) \hat{\mu}(s), \quad \frac{d}{d \hat{s}} \hat{\mu}(s)=0 .
$$

Therefore, from equations $(2.2)$ we obtain that $\hat{\lambda}(s)=\hat{s}+\hat{k}$ for some dual constant $\hat{k}=k+\varepsilon k^{*}$, where $k, k^{*} \in \mathbb{R}$, and that $\hat{\mu}(s)=$ dual constant. On the other hand, since

$$
\hat{\lambda}(s) \hat{\kappa}(s) \neq 0,
$$

the dual constant $\hat{\mu}(s)$ is nonzero. Taking into consideration equation (2.1), we have

$$
\langle\hat{x}(\vec{s}), \hat{t}(s)\rangle=\epsilon_{1} \hat{s}+\hat{k} .
$$

Thus, statement (ii) is proved.

Now, differentiating the equation

$$
\hat{\rho}^{2}=|\langle\vec{x}(\vec{s}), \hat{x}(\vec{s})\rangle|
$$

with respect to the dual arc length function $\hat{s}$ and using equation (2.3), we find

$$
\hat{\rho}^{2}=\left|\epsilon_{1} \hat{s}^{2}+\hat{c}_{1} \hat{s}+\hat{c}_{2}\right|,
$$

where $\hat{c}_{1}$ and $\hat{c}_{2}$ are dual constants. Hence, we get statement (i).

From equation (2.1) it follows that the normal component $\hat{x}^{N}$ of the position vector of the dual Lorentzian curve is given by equation

$$
\langle\hat{x}(\vec{s}), \hat{b}(s)\rangle=\epsilon_{3} \hat{\mu}(s) .
$$

Here, since $\hat{\mu}(s)$ is a dual constant, we get that the normal component $\hat{x}^{N}$ of the position vector of the dual Lorentzian curve is of dual constant length. Therefore, statement (iii) is proved.

And finally, from the dual constancy of $\hat{\mu}$, the fact that $\kappa>0$, the relation $\hat{\lambda}(s)=\hat{s}+\hat{k}$, and the second equation of (2.2) we have statement (iv).

Conversely, let us suppose that statement (i) or statement (ii) holds. Then we are of $\langle\hat{x}(\vec{s}), \hat{t}(s)\rangle=\epsilon_{1} \hat{s}+\hat{k}, \hat{k} \in \mathbb{D}$. If we take the derivative of this equation with respect to $\hat{s}$, we get $\hat{\kappa}\langle\hat{x}(s), \hat{n}(s)\rangle=0$. As $\kappa>0$ by assumption, $\langle\hat{x}(s), \hat{n}(s)\rangle=0$ is found. Hence, the dual Lorentzian curve $\hat{x}$ is rectifying.

Now, suppose that statement (iii) holds. Then we obtain

$$
\langle\hat{x}(\vec{s}), \hat{x}(\vec{s})\rangle=\epsilon_{1}\langle\hat{x}(\vec{s}), \hat{t}(s)\rangle^{2}+\hat{c},
$$

where $\hat{c}=\epsilon_{3} \hat{\mu}^{2}(s)$ is a dual constant. Differentiating this equation with respect to $\hat{s}$ we find that

$$
\langle\hat{x} \vec{s}), \vec{t}(s)\rangle=\left(1+\epsilon_{1} \hat{\kappa}\langle\hat{x}(\vec{s}), \hat{n}(s)\rangle\right)\langle\vec{x}(\vec{s}), \vec{t}(s)\rangle .
$$


On the other hand, since the distance function $\hat{\rho}$ is a non-constant, we get $\langle\hat{x}(s), \vec{t}(s)\rangle \neq 0$. Moreover, from equation (2.5) and the assumption that $\kappa>0$, we obtain $\langle\hat{x}(s), \hat{n}(s)\rangle=0$ which means that $\hat{x}$ is a rectifying dual Lorentzian curve.

Finally, if statement (iv) holds, then by using Frenet equations (1.2), we easily obtain that the dual Lorentzian curve $\hat{x}$ is a rectifying curve.

The Frenet formulae given by (1.2) can be interpreted kinematically as follows: If a moving point moves along a dual Lorentzian curve $\hat{x}$ with dual unit speed, then the moving dual frame $\{\vec{t}(s), \hat{n}(s), \hat{b}(s)\}$ moves in accordance with (1.2). This motion consists of an instantaneous translation along $\hat{x}$ and an instantaneous rotation about $\hat{x}$. The an instantaneous rotation is determined by an angular velocity vector given by dual Darboux vector

$$
\overrightarrow{\hat{\partial}}=\epsilon_{1} \epsilon_{2} \hat{\tau} \hat{t}(s)+\epsilon_{1} \epsilon_{3} \hat{\kappa} \hat{b}(s)
$$

which satisfies

$$
\begin{aligned}
\frac{d \vec{t}(s)}{d \hat{s}} & =\overrightarrow{\hat{\partial}} \times \hat{t}(s), \\
\frac{d \hat{n}(s)}{d \hat{s}} & =\overrightarrow{\hat{\partial}} \times \hat{n}(s), \\
\frac{d \hat{b}(s)}{d \hat{s}} & =\overrightarrow{\hat{\partial}} \times \hat{b}(s) .
\end{aligned}
$$

The direction of the dual Darboux vector is that of the instantaneous axis of rotation. Dual Darboux rotation of dual Frenet frame can be separated into two rotation motions, see [1] and [12]:

Dual tangent vector $\vec{t}(s)$ rotates with a $\hat{\kappa}$ angular speed round dual binormal vector $\vec{b}(s)$, that is

$$
\frac{d \hat{t}(s)}{d \hat{s}}=\left(\epsilon_{1} \epsilon_{3} \hat{\kappa} \hat{b}(s)\right) \times \vec{t}(s)
$$

and dual binormal vector $\hat{b}(s)$ rotates with a $\hat{\tau}$ angular speed round dual tangent vector $\hat{t}(s)$, that is

$$
\frac{d \hat{b}(s)}{d \hat{s}}=\left(\epsilon_{1} \epsilon_{2} \hat{\tau} \hat{t}(s)\right) \times \vec{b}(s) .
$$

By applying the second equation of (2.2), we know that the position vector of a rectifying dual Lorentzian curve is always in the direction of the dual Darboux vector (see [4]). Therefore, rectifying dual Lorentzian curves can be interpreted kinematically as those dual Lorentzian curves whose position vector field determines the axis of instantaneous rotation at each point of the dual Lorentzian curve. 
Now, for rectifying dual Lorentzian curves we shall give the following characterization in terms of the harmonic curvature $\frac{\hat{\tau}}{\hat{\kappa}}$.

Theorem 2.2. Let $\hat{x}: I \rightarrow \mathbb{D}_{1}^{3}$ be a non-null dual Lorentzian curve with $\hat{\kappa}=\kappa+\varepsilon \kappa^{*}$ such that $\kappa>0$ and let $\hat{s}$ be dual arc length of $\hat{x}$. Then $\hat{x}$ is congruent to a rectifying dual Lorentzian curve if and only if its the harmonic curvature $\frac{\hat{\tau}}{\hat{\kappa}}$ is a non-constant linear function in dual arc length function $\hat{s}$, that $i s, \frac{\hat{\tau}}{\hat{\kappa}}=\hat{c}_{1} \hat{s}+\hat{c}_{2}$, where $\hat{c}_{1}=c_{1}+\varepsilon c_{1}^{*}, \hat{c}_{2}=c_{2}+\varepsilon c_{2}^{*} \in \mathbb{D}$ and $c_{1} \neq 0$.

Proof. Let $\hat{x}: I \rightarrow \mathbb{D}_{1}^{3}$ be a unit speed dual Lorentzian curve with $\hat{\kappa}=\kappa+\varepsilon \kappa^{*}$, which is $\kappa>0$. If $\hat{x}$ is a rectifying dual Lorentzian curve, then by the proof of Theorem 2.1 and by relation $(2.2)$, we get

$$
\frac{\hat{\tau}}{\hat{\kappa}}=\epsilon_{2} \epsilon_{3} \frac{\hat{s}+\hat{k}}{\hat{a}},
$$

where $\hat{a}$ and $\hat{k}$ are dual constants. Hence, the harmonic curvature $\frac{\hat{\tau}}{\hat{\kappa}}$ of the dual Lorentzian curve $\hat{x}$ is a non-constant linear function of the dual arc length function $\hat{s}$.

Conversely, let us suppose that the harmonic curvature $\frac{\hat{\tau}}{\hat{\kappa}}$ is a non-constant linear function such that $\frac{\hat{\tau}}{\hat{\kappa}}=\hat{c}_{1} \hat{s}+\hat{c}_{2}$, where $\hat{c}_{1}, \hat{c}_{2} \in \mathbb{D}$ and $\hat{c}_{1}$ is nowhere pure dual. If we take $\hat{a}=\frac{\epsilon_{2} \epsilon_{3}}{\hat{c}_{1}}$ and $\hat{k}=\epsilon_{2} \epsilon_{3} \hat{a} \hat{c}_{2}$, then we have $\frac{\hat{\tau}}{\hat{\kappa}}=\frac{\hat{s}+\hat{k}}{\epsilon_{2} \epsilon_{3} \hat{a}}$. Therefore, by using Frenet equations (1.2), we get that

$$
\frac{d}{d \hat{s}}[\hat{x}(\vec{s})-(\hat{s}+\hat{k}) \hat{t}(s)-\hat{a} \hat{b}(s)]=0,
$$

which means that the dual Lorentzian curve $\hat{x}$ is congruent to a rectifying dual Lorentzian curve.

\section{Classification of rectifying dual Lorentzian curves}

Now, we shall give a theorem determining the parameterization of unit speed rectifying curves in $\mathbb{D}_{1}^{3}$.

Theorem 3.1. Let $\hat{x}: I \rightarrow \mathbb{D}_{1}^{3}$ be a non-null dual Lorentzian curve with $\hat{\kappa}=\kappa+\varepsilon \kappa^{*}$ such that $\kappa>0$. Then,

(i) $\hat{x}$ is a rectifying dual Lorentzian curve with a spacelike rectifying plane if and only if, up to parameterization, $\hat{x}$ is given by

$$
\hat{x}(t)=(\hat{a} \sec \hat{t}) \hat{y}(t),
$$

where $\hat{a}=a+\varepsilon a^{*}(a>0)$ is a dual number and $\hat{y}=\hat{y}(t)$ is a dual unit speed spacelike curve in pseudo dual sphere $\mathbb{S}_{1}^{2}(1)$.

(ii) $\hat{x}$ is a spacelike (timelike) rectifying dual Lorentzian curve with a timelike rectifying plane and a spacelike (timelike) position vector if and only if

$$
\hat{x}(t)=(\hat{a} \operatorname{cosech} \hat{t}) \hat{y}(t),
$$

where $\hat{y}(t)$ is a dual unit speed timelike (spacelike) curve lying in the pseudo dual sphere $\mathbb{S}_{1}^{2}(1)$ (pseudo dual hyperbolic space $\mathbb{H}_{0}^{2}(1)$ ). 
(iii) $\hat{x}$ is a spacelike (timelike) rectifying dual Lorentzian curve with a timelike rectifying plane and a timelike (spacelike) position vector if and only if, up to a parametrization, $\hat{x}$ is given by

$$
\hat{x}(t)=(\hat{a} \operatorname{sech} \hat{t}) \hat{y}(t),
$$

where $\hat{y}(t)$ is a dual unit speed spacelike (timelike) curve lying in the pseudo dual hyperbolic space $\mathbb{H}_{0}^{2}(1)$ (pseudo dual sphere $\mathbb{S}_{1}^{2}(1)$ ).

Proof. (i) Let us suppose that the dual Lorentzian curve $\hat{x}$ is a dual unit speed non-null rectifying dual curve with spacelike rectifying plane in $\mathbb{D}_{1}^{3}$. Since the position vector lies in the spacelike rectifying plane, we get $\langle\hat{x}(s), \hat{x}(s)\rangle>0$ and $\langle\overrightarrow{\hat{t}}, \overrightarrow{\hat{t}}\rangle=\langle\overrightarrow{\hat{b}}, \overrightarrow{\hat{b}}\rangle=1$. From Theorem 2.1, we know that the dual distance function $\hat{\rho}=\|\hat{x} \overrightarrow{(s)}\|$ satisfies $\hat{\rho}^{2}=\hat{s}^{2}+\hat{c}_{1} \hat{s}+\hat{c}_{2}$ for some dual constants $\hat{c}_{1}$ and $\hat{c}_{2}$. After making a suitable translation in $\hat{s}$, we have $\hat{\rho}^{2}=\hat{s}^{2}+\hat{c}$ for some dual constant $\hat{c}=c+\varepsilon c^{*}$, where $c>0$. Therefore, we may choose $\hat{c}=\hat{a}^{2}$. Next, we define a dual Lorentzian curve $\hat{y}$ lying in the pseudo dual sphere $\mathbb{S}_{1}^{2}(1)$ by $\hat{y}=\frac{\hat{x}}{\hat{\rho}}$. Hence, we obtain

$$
\hat{x}(s)=\sqrt{\hat{s}^{2}+\hat{a}^{2}} \hat{y}(s) .
$$

Differentiating equation (3.2) with respect to $\hat{s}$, we get

$$
\frac{d \hat{x}(s)}{d \hat{s}}=\frac{\hat{s}}{\sqrt{\hat{s}^{2}+\hat{a}^{2}}} \hat{y}(s)+\sqrt{\hat{s}^{2}+\hat{a}^{2}} \frac{d \hat{y}(s)}{d \hat{s}} .
$$

On the other hand, since $\langle\hat{y}(s), \hat{y}(s)\rangle=1$, it follows that $\left\langle\hat{y}(s), \frac{d \hat{y}(s)}{d \hat{s}}\right\rangle=0$. Thus, if we take into consideration that $\hat{x}=\hat{x}(s)$ is a dual unit speed non-null rectifying dual Lorentzian curve in (3.3), we find $\left\|\frac{d \hat{y}(s)}{d \hat{s}}\right\|=\frac{\hat{a}}{\hat{s}^{2}+\hat{a}^{2}}$. Now, let us put

$$
\hat{t}=\int_{0}^{\hat{s}} \frac{\hat{a}}{\hat{u}^{2}+\hat{a}^{2}} d \hat{u}=\arctan \left(\frac{\hat{s}}{\hat{a}}\right),
$$

which is the dual arc length parameter of the curve $\hat{y}$. So, $\hat{s}=\hat{a} \tan \hat{t}$. By using this in (3.2), we get (3.1).

Conversely, let us suppose that $\hat{x}: I \rightarrow \mathbb{D}_{1}^{3}$ is a dual Lorentzian curve defined by

$$
\hat{x}(t)=(\hat{a} \sec \hat{t}) \hat{y}(t)
$$

for a dual number $\hat{a}=a+\varepsilon a^{*}(a>0)$, where $\hat{y}=\hat{y}(t)$ is a dual unit speed spacelike curve lying in the pseudo dual sphere $\mathbb{S}_{1}^{2}(1)$. Differentiating equation (3.5) with respect to $\hat{t}$, we obtain

$$
\frac{d \hat{x}(t)}{d \hat{t}}=(\hat{a} \sec \hat{t})\left\{(\tan \hat{t}) \hat{y}(t)+\frac{d \hat{y}(t)}{d \hat{t}}\right\} .
$$


Since $\hat{y}(t)$ and $\frac{d \hat{y}(t)}{d \hat{t}}$ are orthonormal vector fields, from (3.6),

$$
\left\|\frac{d \hat{x}(t)}{d \hat{t}}\right\|=\hat{a} \sec ^{2} \hat{t}
$$

is found. Equations (3.5), (3.6), and (3.7) mean that

$$
\left\langle\hat{x}^{N}, \hat{x}^{N}\right\rangle=\hat{\rho}^{2}(t)-\frac{\left\langle\hat{x}(t), \frac{d \hat{x}(t)}{d \hat{t}}\right\rangle^{2}}{\left\|\frac{d \hat{x}(t)}{d \hat{t}}\right\|^{2}}=\hat{a}^{2} .
$$

And this shows that the normal component $\hat{x}^{N}$ of the position vector has dual constant length. Therefore, from Theorem 2.1 it is figure out that $\hat{x}$ is a rectifying dual Lorentzian curve.

(ii) Let us first suppose that $\hat{x}$ is a spacelike rectifying dual curve with a timelike rectifying plane and a spacelike position vector. Then, we obtain

$$
\langle\hat{x}(\vec{s}), \hat{x} \overrightarrow{(s)}\rangle>0, \quad \epsilon_{1}=\langle\vec{t}(s), \hat{t}(s)\rangle=1, \text { and } \epsilon_{2}=\langle\hat{b}(s), \hat{b}(s)\rangle=-1 .
$$

From proof of Theorem 2.1, we get $\hat{\rho}^{2}=(\hat{s}+\hat{c})^{2}-\hat{a}^{2}$, where $\hat{a}$ and $\hat{c}$ are dual constants. We may apply a translation with respect to $\hat{s}$ such that $\hat{\rho}^{2}=\hat{s}^{2}-\hat{a}^{2}$, $|s|>a$. Now, if we define a dual curve $\hat{y}(s)$ lying in the pseudo dual sphere $\mathbb{S}_{1}^{2}(1)$ by

then we find

$$
\hat{y}(s)=\frac{\hat{x}(s)}{\hat{\rho}(s)}
$$

$$
\hat{x}(s)=\sqrt{\hat{s}^{2}-\hat{a}^{2}} \hat{y}(s) .
$$

By taking differentiation of the previous equation with respect to $\hat{s}$, we have

$$
\frac{d \hat{x}(s)}{d \hat{s}}=\frac{\hat{s}}{\sqrt{\hat{s}^{2}-\hat{a}^{2}}} \hat{y}(s)+\sqrt{\hat{s}^{2}-\hat{a}^{2}} \frac{d \hat{y}(s)}{d \hat{s}} .
$$

Since $\left\langle\hat{y}(s), \frac{d \hat{y}(s)}{d \hat{s}}\right\rangle=0$,

$$
\left\langle\frac{d \hat{x}(s)}{d \hat{s}}, \frac{d \hat{x}(s)}{d \hat{s}}\right\rangle=\frac{\hat{s}^{2}}{\hat{s}^{2}-\hat{a}^{2}}+\left(\hat{s}^{2}-\hat{a}^{2}\right)\left\langle\frac{d \hat{y}(s)}{d \hat{s}}, \frac{d \hat{y}(s)}{d \hat{s}}\right\rangle=1
$$

is found. From the last equation, we have

$$
\left\langle\frac{d \hat{y}(s)}{d \hat{s}}, \frac{d \hat{y}(s)}{d \hat{s}}\right\rangle=-\frac{\hat{a}^{2}}{\left(\hat{s}^{2}-\hat{a}^{2}\right)^{2}},
$$

which means that $\hat{y}$ is a timelike dual curve. By using (3.9), we easily get that $\left\|\frac{d \hat{y}(s)}{d \hat{s}}\right\|=\frac{\hat{a}}{\hat{s}^{2}-\hat{a}^{2}},|s|>a$. Let

$$
\hat{t}=\int_{0}^{\hat{s}}\left\|\frac{d \hat{y}(\hat{u})}{d \hat{u}}\right\| d \hat{u}
$$


be the arc length parameter of the dual curve $\hat{y}$. Then we obtain $\hat{t}=\int_{0}^{\hat{s}} \frac{\hat{a}}{\hat{u}^{2}-\hat{a}^{2}} d \hat{u}$, and thus $\hat{t}=-\operatorname{coth}^{-1}\left(\frac{\hat{s}}{\hat{a}}\right)$ or $\hat{s}=-\hat{a} \operatorname{coth}(\hat{t})$. Substituting this into equation (3.8), we have

$$
\hat{x}(t)=(\hat{a} \operatorname{cosech} \hat{t}) \hat{y}(t) .
$$

Conversely, let us assume that dual curve $\hat{x}(t)$ is $\hat{x}(t)=(\hat{a} \operatorname{cosech} \hat{t}) \hat{y}(t)$, where $\hat{y}(t)$ is a dual unit speed timelike curve lying in the pseudo dual sphere $\mathbb{S}_{1}^{2}(1)$. Differentiating the equation $\hat{x}(t)=(\hat{a} \operatorname{cosech} \hat{t}) \hat{y}(t)$ with respect to $\hat{t}$, we get

$$
\frac{d \hat{x}(t)}{d \hat{t}}=\frac{\hat{a}}{\sinh ^{2} \hat{t}}\left(\frac{d \hat{y}(t)}{d \hat{t}} \sinh \hat{t}-\hat{y}(t) \cosh \hat{t}\right)
$$

Taking into consideration equation (3.10), we have

$$
\left\langle\hat{x}(t), \frac{d \hat{x}(t)}{d \hat{t}}\right\rangle=-\frac{\hat{a}^{2} \cosh \hat{t}}{\sinh ^{3} \hat{t}} \text { and }\left\langle\frac{d \hat{x}(t)}{d \hat{t}}, \frac{d \hat{x}(t)}{d \hat{t}}\right\rangle=\frac{\hat{a}^{2}}{\sinh ^{4} \hat{t}} .
$$

Therefore, it happens $\left\|\frac{d \overrightarrow{\hat{x}(t)}}{d \hat{t}}\right\|=\frac{\hat{a}}{\sinh ^{2} \hat{t}}$. Now, let us put $\hat{x}(t)=\hat{\gamma}(t) \frac{d \overrightarrow{\hat{x}(t)}}{d \hat{t}}+\hat{x}^{N}$, where $\hat{\gamma}(t) \in \mathbb{D}$, and $\hat{x}^{N}$ is a normal component of the position vector $\hat{x}(t)$. Then, we obtain that $\hat{\gamma}(t)=\frac{\left\langle\hat{x}(t), \frac{d \overrightarrow{\hat{x}}(t)}{d \hat{t}}\right\rangle}{\left\langle\hat{x}^{\prime}(t), \frac{d \vec{x}(t)}{d t}\right\rangle}$ and hence

$$
\left\langle\hat{x}^{N}, \hat{x}^{N}\right\rangle=\langle\hat{x}(t), \hat{x}(t)\rangle-\frac{\left\langle\hat{x}(t), \frac{d \vec{x}(t)}{d \hat{t}}\right\rangle^{2}}{\left\langle\frac{d \vec{x}(t)}{d \hat{t}}, \frac{d \vec{x}(t)}{d \hat{t}}\right\rangle} .
$$

Since $\langle\hat{x}(t), \vec{x}(t)\rangle=\frac{\hat{a}^{2}}{\sinh ^{2} \hat{t}}$ and by using (3.11), equation (3.12) becomes

$$
\left\langle\hat{x}^{N}, \hat{x}^{N}\right\rangle=-\hat{a}^{2}=\text { dual constant. }
$$

Thus, $\left\|\hat{x}^{N}\right\|=$ dual constant and $\hat{\rho}=\frac{\hat{a}}{\sinh \hat{t}} \neq$ dual constant, from Theorem 2.1 this means that the curve $\hat{x}$ is rectifying.

The proof in the case when $\hat{x}$ is a timelike rectifying dual curve with a timelike rectifying plane and a timelike position vector is analogous.

iii) The proof is analogous to the proofs of the statements (i) and (ii).

The following corollary given for non-null dual curves gives a link between our work and the classical surface theory.

Corollary 3.2. (1) Let $\hat{x}$ be a dual rectifying curve lying in spacelike rectifying plane. Then

$$
\frac{1}{\hat{a} \sec \hat{t}} \hat{x}(t)
$$

corresponds to timelike ruled surface in $\mathbb{R}_{1}^{3}$. 
(2) Let $\hat{x}$ be a spacelike (timelike) dual rectifying curve with spacelike (timelike) position vector lying in timelike rectifying plane. Then

$$
\frac{1}{\hat{a} \operatorname{cosech} \hat{t}} \hat{x}(t)
$$

corresponds to spacelike (timelike) ruled surface in $\mathbb{R}_{1}^{3}$.

(3) Let $\hat{x}$ be a spacelike (timelike) dual rectifying curve with timelike (spacelike) position vector lying in timelike rectifying plane. Then

$$
\frac{1}{\hat{a} \operatorname{sech} \hat{t}} \hat{x}(t)
$$

corresponds to timelike (spacelike) ruled surface in $\mathbb{R}_{1}^{3}$.

Acknowledgment. The authors would like to thanks A. Yücesan and N. Ayyıldız for their suggestions.

\section{References}

[1] N. Ayyıldız, A. C. Çöken, and A. Yücesan, On the dual Darboux rotation axis of the spacelike dual space curve, Demonstratio Math. 37 (2004), no. 1, 197-202.

[2] _ A characterization of dual Lorentzian spherical curves in the dual Lorentzian space, Taiwanese J. Math. 11 (2007), no. 4, 999-1018.

[3] B. Y. Chen, When does the position vector of a space curve always lie in its rectifying plane?, Amer. Math. Monthly 110 (2003), no. 2, 147-152.

[4] B. Y. Chen and F. Dillen, Rectifying curves as centrodes and extremal curves, Bull. Inst. Math. Acad. Sinica 33 (2005), no. 2, 77-90

[5] H. W. Guggenheimer, Differential Geometry, McGraw-Hill, New York, 1963.

[6] K. İlarslan, E. Nesovic, and M. Petrovic, Some characterizations of rectifying curves in the Minkowski 3-space, Novi Sad J. Math. 33 (2003), no. 2, 23-32.

[7] B. O'Neill, Semi-Riemannian geometry with applications to relativity, London: Academic Press, 1983.

[8] H. H. Uğurlu and A. Çalışkan, The study mapping for directed spacelike and timelike lines in Minkowski 3- Space $\mathbb{R}_{1}^{3}$, Mathematical and Computational Applications, 1 (1996), no. 2, 142-148.

[9] G. R. Veldkamp, On the use of dual numbers, vectors and matrices in instantaneous, spatial kinematics, Mechanism and Machine Theory, 11 (1976), no. 2, 141-156.

[10] Y. Yaylı, A. Çalışan, and H. H. Uğurlu, The E. Study maps of circles on dual hyperbolic and Lorentzian unit spheres $H_{0}^{2}$ and $S_{1}^{2}$, Math. Proc. R. Ir. Acad. 102A (2002), no. 1, $37-47$.

[11] A. Yücesan, N. Ayyıldı, and A. C. Çöken, On rectifying dual space curves, Rev. Mat. Complut. 20 (2007), no. 2, 497-506.

[12] A. Yücesan, A. C. Çöken, and N. Ayyıldı, On the dual Darboux rotation axis of the timelike dual space curve, Balkan J. Geom. Appl. 7 (2002), no. 2, 137-142.

EMINE ÖZBEY

Department of Mathematics

Süleyman Demirel University Graduate School of Natural and Sciences 32260-ISPARTA, TURKEY

E-mail address: emineozbey@stud.sdu.edu.tr 
Mehmet Oral

Department of Mathematics

Süleyman Demirel University Graduate School of Natural and Sciences 32260-Isparta, TURKEY

E-mail address: meoral@stud.sdu.edu.tr 